\title{
DEMOGRAPHICS BUSINESS: INTERNET PARA OPERACIONES DE BANCA ELECTRÓNICA Y OTROS SERVICIOS FINANCIEROS. CHIMBOTE, 2017
}

\author{
DEMOGRAPHICS BUSINESS: INTERNET FOR ELECTRONIC \\ BANKING OPERATIONS AND OTHER FINANCIAL SERVICES. \\ CHIMBOTE, 2017
}

\begin{abstract}
Jorge Neciosup Obando ${ }^{1}$, Juan Julio Díaz Espinoza², Carlos González Chavez, Gladys Villarreal Correa ${ }^{4}$
\end{abstract}

\section{RESUMEN}

La presente investigación busca describir los perfiles demográficos de los adultos usuarios de internet que hacen operaciones de banca electrónica y otros servicios financieros en el ámbito de los distritos de Chimbote y Nievo Chimbote. Para ello se ha utilizado la base de datos de la encuesta nacional de INEI, Encuesta Nacional de Hogares del primer trimestre 2017, de ella se ha tomado la sub base correspondiendo a los códigos geo referenciados pertenecientes a los distritos de interés. Los resultados son presentados en tablas de una y doble clasificación y para evaluar la relación con las variables demográficas se aplicó la prueba estadística de independencia de criterios (chi cuadrado). Los resultados nos evidencian que sólo el 9.2\% de adultos de la población en estudio utiliza la banca por internet, como estimación puntual, a lo cual podemos añadir la estimación interválica: con 95\% de confianza afirmamos que los usuarios de internet que utilizan operaciones de banca electrónica y otras operaciones financieras esta-

1 Instituto de Investigación en Población y Desarrollo, Universidad San Pedro, Perú, jorgeneciosup@gmail.com

2 Universidad San Pedro (Cajamarca), Perú juanjuliod@gmail.com

3 Universidad San Pedro, Perú, calologonzalez@yahoo.com

4 Universidad San Pedro, Perú, glavico@hotmail.com 
ría entre 5,8\% y $12,6 \%$. Así mismo se evidencia que existe relación estadísticamente significativa con la edad y el nivel de instrucción de usuarios, correspondiendo mayor uso al grupo de edad 25-44 años con 18,6\%, y al nivel de instrucción superior con 25,4\%. No se advierten diferencias estadísticamente significativas ni por género, ni por estado conyugal. Este estudio permite tener referentes de nichos preferenciales de la utilización de este servicio que moderniza las operaciones financieras.

PALABRAS CLAVE: Uso de internet, operaciones de banca, perfil demográfico.

\section{ABSTRACT}

The present research seeks to describe the demographic profiles of adult Internet users who perform electronic banking and other financial services in the Chimbote and Nuevo Chimbote districts. For this purpose, the database of the INEI national survey, National Household Survey for the first quarter of 2017 has been used, from which the sub base has been taken corresponding to geo referenced codes belonging to the districts of interest. The results are presented in tables of one and double classification and to evaluate the relation with the demographic variables the statistical test of criterion independence (chi squared) was applied. The results show that only $9.2 \%$ of adults in the study population use internet banking as a point estimate, to which we can add the interval estimate: with $95 \%$ confidence, we affirm that Internet users using electronic banking and other financial operations would be between $5.8 \%$ and $12.6 \%$. Likewise, it is evidenced that there is a statistically significant relationship with the age and level of instruction of users, corresponding to greater use in the 25-44 age group with $18.6 \%$, and the higher education level with $25.4 \%$. No statistically significant differences were observed by gender nor by marital status. This study allows to have references of preferential niches of the use of this service that modernizes the financial operations.

KEY WORDS: Internet use, banking operations, demographic profile.

\section{INTRODUCCIÓN}

Si bien la modernidad del uso de internet y con ello las TIC's alcanzó a los hogares, que según el reporte de INEI, el acceso a este servicio en forma domiciliaria pasó de 1,4\% en el 2005 a 16,2\% en el 2015, en la Región Ancash (INEI, 2016); y que independientemente del lugar de uso, domicilio, cabina o centro de trabajo, el uso por edades se reporta en $69,21 \%$ en personas de 19 a 24 años, $43,4 \%$ en aquellas que van de 25 a 40 años, de 26,3\% en las de 41 a 59 años y sólo de 9,0\% en aquellas de 60 y más años. (INEI, 2016). El Diario Gestión, reporta para el año 2016 que el 50,9\% de usuarios de internet lo hace a diario, el 42,5\% con alguna frecuencia semanal, y el restante $6,6 \%$ más esporádicamente. 
Recientemente, el reclamo social en la venta de entradas por internet para el partido de futbol de Perú Colombia, evidenció que no muchos tenían cuenta bancaria y que no muchos efectuaban operaciones financieras por internet.

Este tema no ha sido todavía abordado, así lo exponen estudios europeos inclusive (Fanjul. J. y L. Vandulciel 2009). Los reportes de INEI, dan cuenta del motivo de utilización en temas de información, diversión, comunicación, y sus asociaciones, pero no en la utilización en banca electrónica, probablemente por la todavía escasa frecuencia con que se hace. Por ese motivo nos planteamos el siguiente problema científico.

¿Cuáles son las proporciones de usuarios de internet para operaciones de banca y otros servicios financieros de la población adulta de Chimbote, 2017? y ¿Cómo se relaciona con el perfil demográfico de usuarios edad, sexo, nivel educativo y condición conyugal?

Determinar cuáles son las proporciones de usuarios de internet para operaciones de banca y otros servicios financieros de la población adulta de Chimbote, 2017 y conocer cómo se relacionan con el perfil demográfico de usuarios edad, sexo, nivel de instrucción y condición conyugal.

\section{MATERIAL Y MÉTODOS}

La presente investigación toma un segmento de la base de datos de la Encuesta Nacional ENAHO (Encuesta Nacional de Hogares), dicha sub base tiene como población bajo estudio fue la población adulta con residencia habitual en los distritos de Chimbote y Nuevo Chimbote al primer trimestre del año 2017 y el diseño muestral correspondió a una muestra trietápica de conglomerados urbanos, considerados por INEI en la ronda del primer trimestre 2017 de la Encuesta nacional ENAHO. Dicha muestra comprende un total de 283 adultos, 176 del distrito de Chimbote y 107 del distrito de Nuevo Chimbote.

La información básica fue procesada con auxilio del software IBM-SPSS ver 23, los resultados se presentan en tablas de una y doble clasificación, empleando la prueba de hipótesis de independencia de criterios (Chi cuadrado) para evaluar si existen asociaciones estadísticamente significativas. (Hernández y otros, 2010), (Pineda, 2008)

La significación estadística se valoró a partir del nivel $\mathrm{P}<0,05$. 
Jorge Neciosup Obando, Juan Díaz Espinoza, Carlos González Chavez, Gladys Villarreal Correa

\section{RESULTADOS}

Tabla 1

ADULTOS DE LA MUESTRA SEGÚN CARACTERÍSTICAS DEMOGRÁFICAS. CHIMBOTE Y NUEVO CHIMBOTE, 2017

\begin{tabular}{|c|c|c|}
\hline & $N^{\circ}$ & $\%$ \\
\hline TOTAL & 283 & 100,0 \\
\hline \multicolumn{3}{|l|}{ DISTRITO } \\
\hline Chimbote & 176 & 62,2 \\
\hline Nuevo Chimbote & 107 & 37,8 \\
\hline \multicolumn{3}{|l|}{ GÉNERO } \\
\hline Masculino & 135 & 47,7 \\
\hline Femenino & 148 & 52,3 \\
\hline \multicolumn{3}{|l|}{ EDAD (años) } \\
\hline De 18 a 24 & 38 & 13,4 \\
\hline De 25 a 44 & 70 & 24,7 \\
\hline De 45 a 59 & 61 & 21,6 \\
\hline De 60 a más & 73 & 25,8 \\
\hline Sin dato & 41 & 14,5 \\
\hline \multicolumn{3}{|l|}{ ESTADO CONYUGAL } \\
\hline Soltero (a) & 85 & 30,0 \\
\hline Casado (a) & 103 & 36,4 \\
\hline Conviviente & 29 & 10,2 \\
\hline Otro & 66 & 23,3 \\
\hline \multicolumn{3}{|c|}{ NIVEL DE INSTRUCCIÓN } \\
\hline Hasta primaria & 58 & 20,5 \\
\hline Secundaria & 124 & 43,8 \\
\hline Sup No universitaria & 25 & 8,8 \\
\hline Superior Universitaria & 71 & 25,1 \\
\hline Sin dato & 5 & 1,8 \\
\hline
\end{tabular}

Fuente: ENAHO, 2017

Elaboración: IPODE USP 
Tabla 2

ADULTOS QUE UTILIZAN INTERNET PARA OPERACIONES

DE BANCA Y OTROS SERVICIOS FINANCIEROS.

CHIMBOTE Y NUEVO CHIMBOTE, 2017

\begin{tabular}{ccc}
\hline $\begin{array}{c}\text { Uso de internet para operaciones de } \\
\text { banca y otras actividades financieras }\end{array}$ & $N^{\circ}$ & $\%$ \\
\hline SI & 26 & 9,2 \\
NO & 257 & 90,8 \\
\hline Total & 283 & 100,0 \\
\hline
\end{tabular}

Fuente: ENAHO, 2017

Elaboración: IPODE - USP.

IC 95\% de la proporción de usuarios $\quad 5,8 \%-12,6 \%$

Tabla 3

ADULTOS QUE UTILIZAN INTERNET PARA OPERACIONES DE BANCA Y OTROS SERVICIOS FINANCIEROS SEGÚN GÉNERO.

CHIMBOTE Y NUEVO CHIMBOTE, 2017

\begin{tabular}{|c|c|c|c|c|c|c|}
\hline \multirow{3}{*}{$\begin{array}{l}\text { Uso de internet para } \\
\text { Operaciones de banca } \\
\text { y otros serv. Financ. }\end{array}$} & \multicolumn{4}{|c|}{ Género } & \multirow{2}{*}{\multicolumn{2}{|c|}{ Todos }} \\
\hline & \multicolumn{2}{|c|}{ Masculino } & \multicolumn{2}{|c|}{ Femenino } & & \\
\hline & № & $\%$ & № & $\%$ & № & $\%$ \\
\hline SI & 16 & 11,9 & 10 & 6,8 & 26 & 9,2 \\
\hline $\mathrm{NO}$ & 119 & 88,1 & 138 & 93,2 & 257 & 90,8 \\
\hline TOTAL & 135 & 100,0 & 148 & 100,0 & 283 & 100,0 \\
\hline
\end{tabular}

Fuente: ENAHO

Prueba de Independencia de Criterios $\mathrm{X}^{2}=2,197 \quad$ N.S. 
Tabla 4

ADULTOS QUE UTILIZAN INTERNET PARA OPERACIONES DE

BANCA Y OTROS SERVICIOS FINANCIEROS SEGÚN EDAD.

CHIMBOTE Y NUEVO CHIMBOTE, 2017

\begin{tabular}{|c|c|c|c|c|c|c|c|c|}
\hline \multirow{3}{*}{$\begin{array}{l}\text { Uso de internet para } \\
\text { operaciones de banca } \\
\text { y otros serv. Financ. }\end{array}$} & \multicolumn{8}{|c|}{ Edad* } \\
\hline & \multicolumn{2}{|c|}{$18-24 * *$} & \multicolumn{2}{|c|}{$25-44$} & \multicolumn{2}{|c|}{$45-59$} & \multicolumn{2}{|c|}{$60+$} \\
\hline & $N^{o}$ & $\%$ & $N^{o}$ & $\%$ & № & $\%$ & $N^{o}$ & $\%$ \\
\hline SI & 2 & 5,3 & 13 & 18,6 & 7 & 11,5 & 4 & 5,5 \\
\hline $\mathrm{NO}$ & 36 & 94,7 & 57 & 81,4 & 54 & 88,5 & 69 & 94,5 \\
\hline TOTAL & 38 & 100,0 & 70 & 100,0 & 61 & 100,0 & 73 & 100,0 \\
\hline
\end{tabular}

** Excluido para la prueba estadística.

Fuente: ENAHO.

Prueba de Independencia de Criterios $\mathrm{X}^{2}=5,977 \quad \mathrm{P}<0,05$

Tabla 5

ADULTOS QUE UTILIZAN INTERNET PARA OPERACIONES DE BANCA Y OTROS SERVICIOS FINANCIEROS SEGÚN NIVEL DE INSTRUCCIÓN. CHIMBOTE Y NUEVO CHIMBOTE, 2017

\begin{tabular}{lrrrrrrrr}
\hline \multirow{2}{*}{$\begin{array}{l}\text { Uso de internet para } \\
\text { operaciones de banca } \\
\text { y otros serv. Financ. }\end{array}$} & \multicolumn{7}{c}{ Hasta prim* } & \multicolumn{2}{c}{ Secundaria } & Sup. no universit. & \multicolumn{2}{c}{ Universitaria } \\
\cline { 2 - 9 } & No & $\%$ & No & $\%$ & No & $\%$ & № & $\%$ \\
\hline \multirow{2}{*}{ SI } & 0 & 0,0 & 5 & 4,0 & 3 & 12,0 & 18 & 25,4 \\
NO & 58 & 100,0 & 119 & 96,0 & 22 & 88,0 & 53 & 74,6 \\
TOTAL & 38 & 100,0 & 124 & 100,0 & 25 & 100,0 & 71 & 100,0 \\
\hline
\end{tabular}

Fuente: ENAHO.

Prueba de Independencia de Criterios $\mathrm{X}^{2}=31,011 \quad \mathrm{P}<0,01$ 
Tabla 6

ADULTOS QUE UTILIZAN INTERNET PARA OPERACIONES DE

BANCA Y OTROS SERVICIOS FINANCIEROS SEGÚN ESTADO

CONYUGAL. CHIMBOTE Y NUEVO CHIMBOTE, 2017

\begin{tabular}{ccccccccr}
\hline \multirow{2}{*}{$\begin{array}{l}\text { Uso de internet para } \\
\text { operaciones de banca } \\
\text { y otros serv. Financ. }\end{array}$} & \multicolumn{2}{c}{ Soltero } & \multicolumn{2}{c}{ Casado } & Conviviente & \multicolumn{2}{c}{ Otro e.c. } \\
\cline { 2 - 10 } & No & $\%$ & No & $\%$ & No & $\%$ & No & $\%$ \\
\hline \multirow{2}{*}{ SI } & 9 & 10,6 & 9 & 8,7 & 7 & 24,1 & 1 & 1,5 \\
NO & 58 & 68,2 & 94 & 91,3 & 22 & 75,9 & 65 & 98,5 \\
TOTAL & 85 & 100,0 & 103 & 100,0 & 29 & 100,0 & 66 & 100,0 \\
\hline
\end{tabular}

Fuente: ENAHO.

Prueba de Independencia de Criterios $\mathrm{X}^{2}=0,339 \quad$ N.S.

\section{DISCUSIÓN}

Evidentemente el perfil de los usuarios presentado en la tabla 1 nos evidencia como están clasificados los adultos de la muestra según los perfiles demográficos en estudio y como están representados los distritos de Chimbote y Nuevo Chimbote.

La tabla 2 nos refiere que del total muestral de 283 personas, sólo 26 utilizaban operaciones de banca y otras actividades financieras por internet, que porcentualmente corresponden a 9,2\%. El intervalo de confianza que describe la utilización de este servicio, al 95\% de confianza es de 5,8\% a 12,6\%. (Daniel, 1993).

La tabla 3 muestra la clasificación de usuarios según género y se advierte que en los hombres el porcentaje de usuarios es de $11,9 \%$ y en mujeres de $6,8 \%$, sin embargo al aplicar la prueba de independencia de criterios no se advierte que difieran significativamente.

La tabla 4 nos muestra la clasificación de la muestra según utilización en operaciones de banca y edad la prueba estadística de independencia de criterios nos advierte el rechazo de la supuesta independencia, por ello se advierte relación significativa con la edad, así podemos advertir que mientras en el grupo de 18-24 años la proporción de uso de operaciones de banca es de sólo 5,3\%, que para los de 45-59 años el porcentaje es de 11,5\% y en los mayores de 60 y más de sólo 5,5\%, 
en el grupo de 25 a 44 años, el porcentaje de usuarios para estos servicios es de $18,6 \%$.

En la tabla 5 se muestra nuestros resultados según nivel de instrucción y utilización de internet en operaciones de banca, encontrando que ambos criterios no sonindependientes $(\mathrm{P}<0,01)$, evidenciando que a mayor nivel de instrucción el porcentaje de usuarios es mayor, correspondiendo al nivel superior una tasa de uso de $25,4 \%$.

En la tabla 6 de presenta la clasificación de los sujetos de la muestra según utilización del internet en operaciones de banca y el estado conyugal. No se rechaza la independencia entre ambos criterios.

\section{CONCLUSIONES}

1. El porcentaje de usuarios de banca electrónica y otros servicios financieros en $<$ Chimbote y Nuevo Chimbote es de 9,2\% y con $95 \%$ de confianza se ubican entre $5,8 \%$ y $12,6 \%$.

2. Existe diferencia significativa entre las edades de los usuarios de internet en operaciones de banca, correspondiendo una mayor tasa de utilización a los adultos entre 25 y 44 años con 18,6\%

3. Existe diferencia significativa entre los niveles de instrucción de los usuarios de internet en operaciones de banca, correspondiendo una mayor tasa de utilización a los adultos con nivel de instrucción superior con 25,4\%.

4. No se advierten diferencias estadísticamente significativas entre los perfiles demográficos de género ni de estado conyugal.

\section{REFERENCIAS BIBLIOGRÁFICAS}

Daniel, W. (1993). Bioestadística: Base para el análisis de las Ciencias de la Salud. Limusa. México

Fanjul. J. y L. Vandulciel (2009) "Impacto de las nuevas tecnologías en el negocio bancario español. En Investigaciones Europeas de Dirección y Economía de la Empresa. Vol. 15, 1,

Hernández S. Robert, Fernández C. Carlos, Bautista L. Pilar,(2010), Metodología de la Investigación Científica, McGRAW HILL Interamericana Editores S.A. 5a Edición. Empresa Editora El Comercio, Lima. 2009, pp. 81-93.

INEI (2015) "Estadísticas de las tecnologías de información y comunicación en los hogares". Informe técnico 01. Lima.

Pineda Elia y De Alvarado, Eva. (2008), Metodología de la Investigación. 3aEdición Organización Panamericana de la Salud. Washington, DC. 